\title{
GENERAL SOLUTION OF REYNOLDS EQUATION FOR A JOURNAL BEARING OF FINITE WIDTH*
}

\author{
BY \\ L. N. TAO \\ Illinois Institute of Technology
}

1. Introduction. The governing equation of hydrodynamic lubrication, the Reynolds equation, was established in 1886 [1]. Since then many works on the solutions of the equation have been published. For plane slider bearings, where the film thickness of the lubricating fluid is a linear function of the coordinate variable, solutions for both infinite and finite widths have been obtained. Michell [2] and Muskat et al. [3] gave their solutions for finite width bearing in terms of Bessel functions. In journal bearings where the film thickness is not a linear function of the variables, Sommerfeld [4] showed that for the case of infinitely long bearings the generalized Reynolds equation, a partial differential equation, is reduced to an ordinary differential equation. The solution of the problem can be immediately derived by integrations. However, for journal bearings of finite length, all of these investigators mentioned in their papers that they did not succeed in obtaining the general solution for the finite bearing problem. Michell even stated that the exact solution of Reynolds equation is only possible if the viscosity is constant and the film thickness is a linear function of the variables. Following this line of argument, the subsequent works on the solutions of finite journal bearings** are approximations by means of a wide variety of mathematical techniques. The aim of this paper is to establish an exact and complete solution of the Reynolds equation for finite journal bearings.

In Sec. 2 of this paper the Reynolds equation, by suitable transformations of variables, is changed to a comparatively unknown equation, Heun's equation. In Sec. 3 Heun's equation and its function related to the problems are discussed. The convergency of the Heun function is also studied. Then in Sec. 4 the pressure distributions, as well as the eigenvalues and their eigenfunctions, are established. Finally, the load carrying capacity and the coefficient of friction of the bearing are determine in Secs. 5 and 6 respectively.

2. Governing equation. The governing equation of hydrodynamic lubrication [1] is

$$
\frac{\partial}{\partial x}\left[h^{3} \frac{\partial p}{\partial x}\right]+\frac{\partial}{\partial z}\left[h^{3} \frac{\partial p}{\partial z}\right]=6 \mu U \frac{d h}{\partial x},
$$

where $p, h$ and $\mu$ are the pressure, film thickness and viscosity of the lubricating fluid respectively, and $U$ is the relative velocity of bearing surfaces. In journal bearings $x$ and $z$ are taken along the circumferential and axial directions respectively, and the film thickness may be written as

$$
h=c(1+\eta \cos \theta) \quad \text { and } \quad \eta=\frac{e}{c},
$$

**Received March 24, 1958.

*See bibliography cited in [5] and a recent work [6]. 
where $c$ is the radial clearance and $\eta$ the attitude, a ratio of eccentricity $e$ to radial clearance $c$.

Introducing dimensionless quantities

$$
\theta=\frac{x}{r}, \quad Z=\frac{z}{r}, \quad H=\frac{h}{c}=1+\eta \cos \theta, \quad P=\frac{p}{\rho U^{2}},
$$

with $r$ being the radius of the bearing, Equation (2.1) becomes

$$
\frac{\partial}{\partial \theta}\left[(1+\eta \cos \theta)^{3} \frac{\partial P}{\partial \theta}\right]+\frac{\partial}{\partial Z}\left[(1+\eta \cos \theta)^{3} \frac{\partial P}{\partial Z}\right]=\frac{6 v r}{U c^{2}} \frac{d H}{d \theta} \text {. }
$$

The boundary conditions of the problem are

$$
\begin{aligned}
P(-\pi, Z) & =P(\pi, Z), \\
\frac{\partial P(-\pi, Z)}{\partial \theta} & =\frac{\partial P(\pi, Z)}{\partial \theta}, \\
P(\theta,-L / 2) & =0, \\
P(\theta, L / 2) & =0,
\end{aligned}
$$

where $L=l / d$ and $l$ and $d$ are the length and diameter of the bearing. Conditions (2.5) and (2.6) assure the continuity of the pressure and its first derivative along its circumferential direction, while the last two conditions, (2.7) and (2.8), state that the pressure at free ends is atmospheric.

In order to remove the non-homogeneous term in Eq. (2.4), we let

$$
P(\theta, Z)=\zeta(\theta, Z)+\xi(\theta),
$$

where $\xi(\theta)$ satisfies the equation of two dimensional journal bearings of infinite length, i.e.

$$
\frac{d}{d \theta}\left[(1+\eta \cos \theta)^{3} \frac{d \xi}{d \theta}\right]=\frac{6 \nu r}{U c^{2}} \frac{d H}{d \theta}
$$

and its boundary conditions

$$
\begin{aligned}
\xi(-\pi) & =\xi(\pi), \\
\frac{d \xi(-\pi)}{d \delta} & =\frac{d \xi(\pi)}{d \theta} .
\end{aligned}
$$

The solution of Eq. (2.10) with the given boundary conditions (2.11) is [4]

$$
\xi(\theta)=\frac{6 v r \eta}{U c^{2}\left(2+\eta^{2}\right)} \frac{(2+\eta \cos \theta) \sin \theta}{(1+\eta \cos \theta)^{2}} .
$$

The other function $\zeta(\theta, Z)$ satisfies

$$
\frac{\partial}{\partial \theta}\left[(1+\eta \cos \theta)^{3} \frac{\partial \zeta}{\partial \theta}\right]+\frac{\partial}{\partial Z}\left[(1+\eta \cos \theta)^{3} \frac{\partial \zeta}{\partial Z}\right]=0
$$

and the boundary conditions

$$
\begin{gathered}
\zeta(-\pi, Z)=\zeta(\pi, Z), \\
\frac{\partial \zeta(-\pi, Z)}{\partial \theta}=\frac{\partial \zeta(\pi, Z)}{\partial \theta},
\end{gathered}
$$




$$
\begin{aligned}
\zeta(\theta,-L / 2) & =-\xi(\theta), \\
\zeta(\theta, L / 2) & =-\xi(\theta) .
\end{aligned}
$$

Separation of variables

$$
\zeta(\theta, Z)=\phi(\theta) \cdot \psi(Z)
$$

gives

$$
\frac{d^{2} \phi}{d \theta^{2}}-\frac{3 \eta \sin \theta}{1+\eta \cos \theta} \frac{d \phi}{d \theta}+\lambda \phi=0
$$

and

$$
\frac{d^{2} \psi}{d Z^{2}}-\lambda \psi=0
$$

From Sturm-Liouville theory [7], it can be shown that Eq. (2.19) with the given boundary conditions possesses a sequence of solutions corresponding to a sequence of real, nonnegative eigenvalues $\lambda_{i}$.

The solution of Eq. (2.20) is then

$$
\begin{array}{ll}
\lambda_{i} \neq 0, & \psi_{i}=A_{i} \cosh \left(\lambda_{i}\right)^{1 / 2} Z+B_{i} \sinh \left(\lambda_{i}\right)^{1 / 2} Z \\
\lambda_{i}=0, & \psi_{0}=A_{0} Z+B_{0} .
\end{array}
$$

Furthermore by the substitution of

$$
u=\frac{1-\cos \theta}{2}=\sin ^{2} \frac{\theta}{2}
$$

Eq. (2.19) becomes

$$
\begin{aligned}
u(u-1)\left(u-\frac{1+\eta}{2 \eta}\right) \frac{d^{2} \phi}{d u^{2}}+\left[4 u^{2}-\left(4+\frac{1}{2 \eta}\right) u+\frac{1+\eta}{4 \eta}\right] \frac{d \phi}{d u} & \\
& -\lambda\left(u-\frac{1+\eta}{2 \eta}\right) \phi=0 .
\end{aligned}
$$

This is known as Heun's equation [8].

3. Heun's equation. Heun's equation

$$
\begin{aligned}
z(z-1)(z-a) y^{\prime \prime}+\left[(\alpha+\beta+1) z^{2}-\{\alpha+\beta+1\right. & -\delta+a(\gamma+\delta)\} z \\
& +a \gamma] y^{\prime}+\alpha \beta(z-q) y=0,
\end{aligned}
$$

with $a, q, \alpha, \beta, \gamma$ and $\delta$ being arbitrary constants, is closely related to the algebraic form of Lame's equation (if $\gamma=\delta=\frac{1}{2}, \alpha+\beta=\frac{1}{2}$ ). Clearly with $a=q=1$ or $a=q=0$, Eq. (3.1) is reduced to the hypergeometric equation.

With regular singularities at $0,1, a$ and $\infty$, the Riemann's $P$-function of Eq. (3.1) is

$$
P\left\{\begin{array}{ccccc}
0 & 1 & a & \infty \\
0 & 0 & 0 & \alpha & z \\
1-\gamma & 1-\delta & (\gamma+\delta) & -(\alpha+\beta) & \beta
\end{array}\right\} .
$$

Therefore the solutions of Eq. (3.1) for $\gamma$ being non-integer are

$$
y=1+\sum_{1}^{\infty} c_{n} z^{n}
$$


and

$$
y=z^{1-\gamma}\left[1+\sum_{1}^{\infty} c_{n}^{\prime} z^{n}\right]
$$

The first solution is

$$
y=1+\sum_{1}^{\infty} c_{n} z^{n}=F(a, q ; \alpha, \beta, \gamma, \delta ; z),
$$

where $F(a, q ; \alpha, \beta, \gamma, \delta ; z)$ is known as the Heun function with

$$
\begin{gathered}
a \gamma c_{1}=\alpha \beta q \\
2 a^{2} \gamma(\gamma+1) c_{2}=(\alpha \beta q)^{2}+\{(\alpha+\beta+1-\delta)+(\gamma+\delta) a\} \alpha \beta q-a \gamma \alpha \beta
\end{gathered}
$$

and

$$
\begin{aligned}
a(n+1)(\gamma+n) c_{n+1}=[(\alpha+\beta+n-\delta) n+a n(\gamma+\delta+n-1) \\
+\alpha \beta q] c_{n}-[(n-1)(\alpha+\beta+n-1)+\alpha \beta] c_{n-1} .
\end{aligned}
$$

The other solution is

$$
y=z^{1-\gamma}\left[1+\sum_{1}^{\infty} c_{n}^{\prime} z^{n}\right]=z^{1-\gamma} F\left(a, q_{1} ; \alpha-\gamma+1, \beta-\gamma+1,2-\gamma, \delta ; z\right)
$$

with

$$
q_{1}=\left\{q+\frac{1-\gamma}{\alpha \beta}[(\alpha+\beta)+\delta(a-1)+(1-\gamma)]\right\} \frac{\alpha \beta}{(\alpha-\gamma+1)(\beta-\gamma+1)} .
$$

Now to establish the convergence of the function (3.5) we consider the series (the Heun function with argument $z=1$ ),

$$
\sum_{n=0}^{\infty} C_{n}
$$

and the recurrence relation

and

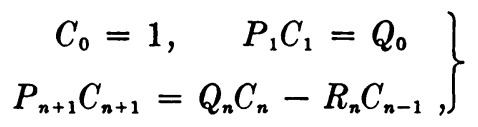

where

$$
\begin{aligned}
& Q_{0}=\alpha \beta q \\
& P_{n}=\frac{a}{n}(\gamma+n-1), \\
& Q_{n}=\frac{1}{n^{2}}[(\alpha+\beta+n-\delta) n+a n(\gamma+\delta+n-1)+\alpha \beta q], \\
& R_{n}=\frac{1}{n^{2}}[(n-1)(\alpha+\beta+n-1)+\alpha \beta] .
\end{aligned}
$$

Let

$$
N_{n}=\frac{C_{n}}{C_{n-1}}
$$


and

$$
P=\lim _{n \rightarrow \infty} P_{n}, \quad Q=\lim _{n \rightarrow \infty} Q_{n}, \quad R=\lim _{n \rightarrow \infty} R_{n}
$$

then the recurrence relation (3.9) becomes

$$
N_{n}=\frac{R_{n}}{Q_{n}-P_{n+1} N_{n+1}} .
$$

By repeating substitutions, Eq. (3.12) may be written as an infinite continued fraction,

$$
N_{n}=\frac{R_{n}}{\mid Q_{n}}-\frac{P_{n+1} R_{n+1} \mid}{\mid Q_{n+1}}-\frac{P_{n+2} R_{n+2} \mid}{\mid Q_{n+2}}-\cdots .
$$

It can be shown [9] that this continued fraction is convergent when the roots $\left(\rho_{1}, \rho_{2}\right)$ of the equation

$$
P \rho^{2}-Q \rho+R=0
$$

are $\left|\rho_{1}\right| \neq\left|\rho_{2}\right|$, or $\rho_{1}=\rho_{2}$ and it is divergent when $\rho_{1} \neq \rho_{2}$ but $\left|\rho_{1}\right|=\left|\rho_{2}\right|$. Also in the convergent case with $\left|\rho_{1}\right|<\left|\rho_{2}\right|$ or $\rho_{1}=\rho_{2}$ we have

$$
\lim _{n \rightarrow \infty} N_{n} \rightarrow \rho_{1} \text {. }
$$

In the present series $P=a, Q=1+a$, and $R=1$, the roots of Eq. (3.14) are 1 and $1 / a$. This, in turn, shows that the Heun function (3.5) is convergent for all $|z|<|a|$ if $|a|>1$ or for all $|z|<1$ if $|a|<1$ or $a=1$.

4. Pressure distribution. By comparison of Eqs. (2.23) and (3.1) we get

$$
\begin{gathered}
a=\frac{1+\eta}{2 \eta}, \quad q=\frac{1+\eta}{2 \eta}, \quad \alpha, \beta=\frac{1}{2}\left[3 \pm(9+4 \lambda)^{1 / 2}\right] \\
\gamma=\frac{1}{2}, \quad \delta=\frac{1}{2} .
\end{gathered}
$$

Since $a>1$ when $\eta<1$, the Heun function of the problem is convergent for all $\theta$. And $a=1$ when $\eta=1$, the Heun function (or the hypergeometric function) is divergent. However, $\eta=1$ implies a contact of bearing surfaces. This is no longer a case of hydrodynamic lubrication. Hence the complete solution of Eq. (2.13) is

$$
\zeta=\phi_{0} \psi_{0}+\sum_{i=1}^{\infty}\left[A_{i} \cosh \left(\lambda_{i}\right)^{1 / 2} Z+B_{i} \sinh \left(\lambda_{i}\right)^{1 / 2} Z\right]\left(C_{i} F_{i}+D_{i} H_{i}\right),
$$

where

$$
\begin{aligned}
F_{i} & =F\left(\frac{1+\eta}{2 \eta}, \frac{1+\eta}{2 \eta} ; \frac{3+\left(9+4 \lambda_{i}\right)^{1 / 2}}{2}, \frac{3-\left(9+4 \lambda_{i}\right)^{1 / 2}}{2}, \frac{1}{2}, \frac{1}{2} ; u\right), \\
H_{i} & =u^{1 / 2} F\left(\frac{1+\eta}{2 \eta}, q_{1} ; \frac{4+\left(9+4 \lambda_{i}\right)^{1 / 2}}{2}, \frac{4-\left(9+4 \lambda_{i}\right)^{1 / 2}}{2}, \frac{3}{2}, \frac{1}{2} ; u\right), \\
q_{1} & =\left(\frac{7}{4}-\lambda_{i}\right)^{-1}\left[\frac{3}{2}+\frac{1+\eta}{2 \eta}\left(\frac{1}{4}-\lambda_{i}\right)\right], \\
\psi_{0} & =A_{0} Z+B_{0} \\
\phi_{0} & =C_{0} \int(1+\eta \cos \theta)^{-3} d \theta+D_{0}
\end{aligned}
$$

and $A_{i}, B_{i}, C_{i}$ and $D_{i}$ are constants. 
Conditions (2.16) and (2.17) indicate that $\zeta$ is an even function of $Z$, so

$$
A_{0}=0 ; \quad B_{i}=0 .
$$

And Eqs. (2.12), (2.16) and (2.17) imply that $\zeta$ is an odd function of $\theta$. Since

$$
u=\sin ^{2} \frac{\theta}{2}
$$

$F_{i}$ and $H_{i}$ are, therefore, even and odd functions of $\theta$ respectively. This gives $C .=0$. From conditions (2.16), (2.17), (2.7) and (2.8) we then get

$$
C_{0}=D_{0}=0 \text {. }
$$

Also conditions (2.14) and (2.15) require

$$
\begin{aligned}
D_{i} H_{i}(\pi) & =0, \\
C_{i} F_{i}^{\prime}(\pi) & =0 .
\end{aligned}
$$

Since $D_{i} \neq 0$,

$$
H_{i}(\pi)=0 .
$$

Now the pressure distribution with $A_{i}$ to be determined is

$$
P(\theta, Z)=H^{*}(\theta)+\sum_{i=1}^{\infty} A_{i} \cosh \left(\lambda_{i}\right)^{1 / 2} Z \cdot H_{i}\left(\lambda_{i} ; \theta\right),
$$

where

$$
H^{*}(\theta)=\xi(\theta)=\frac{6 \nu r \eta(2+\eta \cos \theta) \sin \theta}{U c^{2}\left(2+\eta^{2}\right)(1+\eta \cos \theta)^{2}}
$$

and the eigenvalues $\lambda_{i}$ are the roots of

$$
H(\lambda ; \pi)=0 .
$$

$H_{i}(\theta)$ are orthogonal with respect to the weight function

$$
w(\theta)=(1+\eta \cos \theta)^{3}
$$

over the interval $(-\pi, \pi)$; that is

$$
\int_{-\pi}^{\pi} w(\theta) H_{i}(\theta) H_{i}(\theta) d \theta=0 \text { for } i \neq j
$$

hence

$$
A_{i}=-\frac{\int_{-\pi}^{\pi} w(\theta) H^{*}(\theta) H_{i}(\theta) d \theta}{\cosh \left[\left(\lambda_{i}\right)^{1 / 2} L / 2\right] \int_{-\pi}^{\pi} w(\theta) H_{i}^{2}(\theta) d \theta}
$$

5. Load carrying capacity. Relating the pressure distribution to the load carrying capacity $W$ of the bearing, $W$ is resolved into two components along and perpendicular to the line of centers, 


$$
\begin{aligned}
& W_{1}=\rho U^{2} r^{2} \int_{-L / 2}^{L / 2} \int_{-\pi}^{\pi} P \cos \theta d \theta d Z, \\
& W_{2}=\rho U^{2} r^{2} \int_{-L / 2}^{L / 2} \int_{-\pi}^{\pi} P \sin \theta d \theta d Z .
\end{aligned}
$$

Due to the fact that $P$ is an odd function of $\theta$, we have

$$
W_{1}=0
$$

and

$\frac{W_{2}}{\rho U^{2} r^{2}}=\frac{12 \pi \nu \eta L r}{U c^{2}\left(2+\eta^{2}\right)\left(1-\eta^{2}\right)^{1 / 2}}+\sum_{i=1}^{\infty} \frac{2 A_{i}}{\left(\lambda_{i}\right)^{1 / 2}} \sinh \left[\left(\lambda_{i}\right)^{1 / 2} L / 2\right] \int_{0}^{1} H_{i}\left(\lambda_{i} ; u\right) d u$.

This result indicates that the line of centers is perpendicular to the load line. The same result has been obtained for the infinitely long journal bearing. With $A_{i}$ always negative in the range $0 \leq \theta \leq \pi$, the last term of Eq. (5.3) is therefore always negative. This implies that the load capacity of finite length bearing is less than that of infinite length bearing. This result has also been found experimentally by electric analogy [10] and theoretically by approximation methods $[3,6]$.

6. Coefficient of friction. The frictional force acting on a journal bearing may be expressed by

$$
F=r^{2} \int_{-L / 2}^{L / 2} \int_{-\pi}^{\pi}\left[\frac{\mu I^{*}}{c(1+\eta \cos \theta)}+\frac{c \rho U^{2}}{2 r}(1+\eta \cos \theta) \frac{\partial P}{\partial \theta}\right] d \theta d Z .
$$

By Eq. (5.3) we obtain

$$
F=\frac{2 \pi \mu U r^{2} L}{c\left(1-\eta^{2}\right)^{1 / 2}}+\frac{c \eta}{2 r} W_{2}
$$

The coefficient of friction is defined as the ratio of fractional force to the total load $W$. And $W_{1}=0$, we have

$$
f=\frac{F}{W}=\frac{2 \pi \mu U r^{2} L}{c\left(1-\eta^{2}\right)^{1 / 2} W_{2}}+\frac{c \eta}{2 r}
$$

\section{BiBLIOGRAPHY}

1. O. Reynolds, "On the theory of lubrication," Phil. Trans. Roy. Soc. London, Ser. A, 177, 157-234 (1886)

2. A. G. M. Michell. "The lubrication of plane surface," Z. Math. u. Phys. 52, 123-137 (1905)

3. (a) M. Muskat, F. Morgan and M. W. Meres, "T'he lubrication of plane sliders of finite width," J. Appl. Phy. 11, 208-219 (1940); (b) M. Muskat and F. Morgan, "The theory of the thick film lubrication of a complete journal bearing of finite length," J. Appl. Phy. 9, 393-409 (1938)

4. A. Sommerfeld, "Zur Hydrodynamischen Theorie der Schmiermittel Reibung," Z. Math. u. Phys. 50, 97-153 (1904)

5. M. C. Shaw and E. F. Macks, "Analysis and lubrication of bearings," McGraw-Hill Book Co., New York, 1949, p. 242

6. M. J. Jacobson, A. Charnes and E. Saibel, "The complete journal bearing with circumferential oil inlet," Trans. ASME 77, 1179-1183 (1955)

7. E. L. Ince, "Ordinary differential equations," Dover Publ., New York, 1944, Chap. 10 
8. (a) E. T. Whittaker and G. N. Watson, " $A$ course of modern analysis," 4th ed., Cambridge Univ. Press, London, 1952, pp. 576-577; (b) A. Erdelyi (Ed.), "Higher transcendental functions," vol. III, McGraw-Hill Book Co., New York, 1955, pp. 57-62

9. O. Perron, "Die Lehre von den Kettenbruchen," Chelsea Publ. Co., New York, 1950, Kap. 7

10. A. Kingsbury, "On problems on the theory of fluid film lubrication with an experimental method of solution," Trans. ASME 53, 59-75 (1931) 